\title{
Primary Disseminated Hydatid Disease Involving Multiple Organs in a Pediatric Patient: A Rare Case Report
}

\author{
Nishu Gupta1® Gunjan Jindal ${ }^{2}$ Hanish Singla ${ }^{3}$ Deepak Garg ${ }^{4}$ \\ ${ }^{1}$ Department of Pediatrics, PGIMER Outreach Center, Sangrur, \\ Punjab, India \\ 2 Department of Radiodiagnosis, Maharishi Markandeshwar Institute \\ Of Medical Sciences and Research, Haryana, India \\ ${ }^{3}$ Department of Radiodiagnosis, Sri Guru Ram Das Institute of \\ Medical Sciences and Research, Amritsar, Punjab, India \\ ${ }^{4}$ Department of Radiodiagnosis, Government Medical College, \\ Chandigarh, India \\ Address for correspondence Nishu Gupta, MD, Department of \\ Pediatrics, PGIMER Satellite Centre, Sangrur, Punjab 148001, India \\ (e-mail: nishigupta.medico@gmail.com). \\ J Child Sci 2021;11:e170-e172.
}

\begin{abstract}
Keywords

- disseminated

- zoonosis

- hydatid disease

Hydatid disease is a parasitic infestation by Echinococcus genus of tapeworm, resulting from ingestion of tapeworm eggs and leading to formation of cysts predominantly in the liver and lungs. Multiorgan involvement in children is unusual. We present a case of a 12-year-old female with multiple organ involvement. Patient had history of abdominal pain, breathlessness, irritability and vomiting. Plain radiograph showed a large ovoid calcific lesion projected over the liver. Abdominal sonography demonstrated multiple hydatid cysts in the liver, spleen and left kidney. Ultrasound findings were further confirmed by contrast-enhanced computed tomography that showed additional hydatid cysts in bilateral lungs. All accessible cysts were excised on laparotomy. Cystic hydatid disease should be suspected in all pediatric patients with multiple cystic lesions, especially in endemic areas.
\end{abstract}

\section{Introduction}

Hydatid disease is the most widespread zoonosis caused by tapeworm of genus Echinococcus granulosus and E. multilocularis. While dogs are definitive hosts, sheep, cattle, goats, or humans may act as accidental intermediate hosts. ${ }^{1} \mathrm{Em}$ bryos released from eggs in the duodenum pass through the mucosa to reach the liver through the portal venous system. Most of the embryos remain trapped in the liver (50-70\%), though the lungs $(20-30 \%)$, kidneys $(2-3 \%)$, spleen $(0.8 \%)$, central nervous system $(0.5-2 \%)$, and bone $(<10 \%)$ may become secondarily involved. ${ }^{2-4}$

We report an atypical case of a 12-year-old female with hydatid cysts involving the liver, spleen, kidney, and para-

received

October 26, 2020

accepted after revision

April 19, 2021 spinal muscles simultaneously. Liver is the most common site of involvement in adults. However, in over $60 \%$ of children, lung is the most frequent site of disease and less than $10 \%$ have concurrent hepatic disease. ${ }^{4}$ Multiple cysts occur in only $30 \%$ of cases and bilateral pulmonary cysts are rare. Renal involvement is also infrequent in pediatric disseminated disease, constituting only $1.9 \%$ of all cases. ${ }^{4}$

\section{Case Report}

A 12-year-old female was admitted to the Pediatrics Department of Shri Guru Ram Das Institute of Medical Sciences, Amritsar in Northern India with complaints of abdominal pain, irritability, vomiting, and prolonged cough in the past

\section{(c) 2021. The Author(s).}

This is an open access article published by Thieme under the terms of the Creative Commons Attribution License, permitting unrestricted use, distribution, and reproduction so long as the original work is properly cited. (https://creativecommons.org/licenses/by/4.0/)

Georg Thieme Verlag KG, Rüdigerstraße 14, 70469 Stuttgart, Germany 


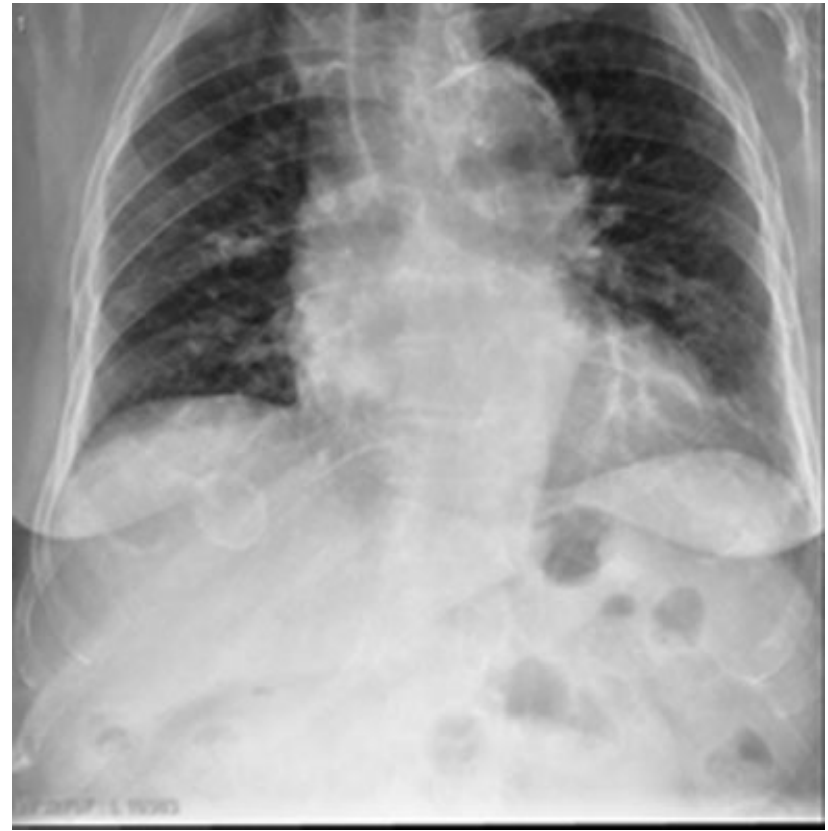

Fig. 1 Plain radiograph shows a large ovoid calcific lesion projected over the liver.

1 year. No history of tuberculosis contact could be elicited. Routine blood tests, erythrocyte sedimentation rate, liver and renal function tests, and urinalysis were normal. Patient came from a rural Indian background where cattle rearing is common practice. At presentation, she had a temperature of $36.4^{\circ} \mathrm{C}$, blood pressure of $110 / 86 \mathrm{~mm} \mathrm{Hg}$, pulse rate of 84 beats per minute, and respiratory rate of 38 breaths per min. Abdominal examination revealed mild right upper quadrant abdominal tenderness and hepatosplenomegaly. Rest of the systemic examination was normal.

Laboratory investigations revealed eosinophilia (18\%) and the enzyme-linked immunosorbent assay for antibody against $E$. granulosus was positive. Plain radiograph showed a large ovoid calcific lesion (calcified pericyst) projecting over the liver (-Fig. 1). On ultrasonography of the abdomen, liver was enlarged measuring $22 \mathrm{~cm}$ in size in mid-clavicular line. Three well-defined round to oval thin-walled anechoic cystic lesions were seen scattered in both lobes of liver (Type 1 Gharbi classification). No evidence of calcification or hemorrhage was seen within the lesion. One of the cysts showed few echogenic membranes floating within it (Type 2 Gharbi classification), another showed fluid collection with septations (Type 3 Gharbi classification), while one cyst was heterogeneous in echotexture (Type 4 Gharbi classification). Cystic lesion was also seen in the splenic parenchyma at its upper pole, which showed a thin strand within it (Type 2 Gharbi classification). Two cystic lesions were also noted at the upper pole of left kidney (Type 1 and 2 Gharbi classification); one of the cysts appeared to be communicating with the upper pole pelvicalyceal system. Mild hydronephrosis with dilated upper ureter was also seen. On contrast-enhanced computed tomography (CECT) abdomen, ultrasound findings were confirmed. Mild amount of subcapsular fluid collection was noted (-Fig. 2). Multiple cystic lesions were

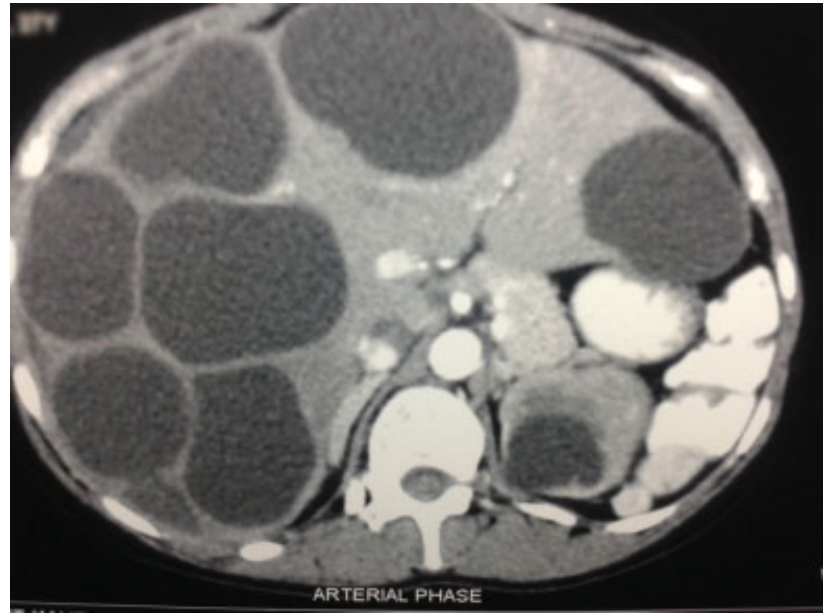

Fig. 2 On contrast-enhanced computed tomography, ultrasound findings were confirmed. All the cystic lesions showed fluid density. The floating membranes and cyst walls show mild degree of enhancement on postcontrast images. Left kidney shows few similar cystic lesions and mild hydronephrosis with dilated upper ureter with abrupt tapering. No calculus was seen in the ureter.

also noted in both lung fields, largely on the right side (Type 1 and 2 Gharbi classification) (-Fig. 3).

On the basis of the above findings, a provisional diagnosis of hydatid cyst with multiple organ dissemination was made. Exploratory laparotomy was performed in view of compressive symptoms caused by the cysts and all accessible cysts were removed. Medical therapy with albendazole ( $400 \mathrm{mg}$ q12h) and praziquantel $(20-75 \mathrm{mg} / \mathrm{kg} /$ day $)$ was continued for 1 year without any significant complications. The child was regularly followed up at 6 monthly intervals for 2 years, with no clinical, serological, or radiological signs of recurrence.

\section{Discussion}

Although hydatid disease is widely endemic in the developing countries in South Asia region, ${ }^{4}$ there are only few

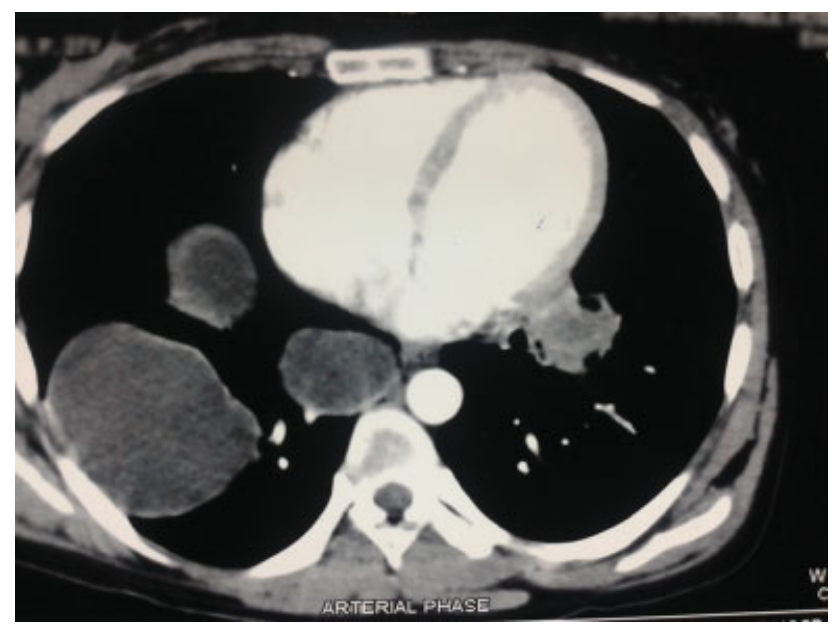

Fig. 3 Multiple cystic lesions in the visualized part of both lung fields more so on the right side. 
reports on clinical characteristics and appropriate treatment of this disease during childhood. ${ }^{5,6}$ Multiple organ dissemination with hydatid cysts rarely occurs in pediatric patients even in endemic areas. ${ }^{7,8}$

The atypical presentation of our case is demonstrated by the numerous and bilateral cysts in the lungs along with hepatic, renal, and paraspinal muscle involvement.

The cysts were classified according to Gharbi classification as:

- Type 1-Pure cystic fluid collection

- Type 2-Fluid collection with membrane separation

- Type 3-Fluid collection with septa

- Type 4-Heterogenous pattern

- Type 5-Completely calcified

Diagnosis can be easily made by ultrasonography and abdominal CECT. ${ }^{7,9}$ CECT scan is advantageous over ultrasound as it is more sensitive and accurate, and can easily detect calcifications, daughter cysts, and hydatid sand. ${ }^{7,9}$

Possible routes of primary hydatid infection of spleen include arterial route, venous route or through systemic dissemination or intraperitoneal spread following ruptured hepatic hydatid cyst. ${ }^{10,11}$

Management of disseminated echinococcus requires a multidisciplinary approach including albendazole therapy, surgery, and PAIR (puncture, aspiration, injection, and respiration). ${ }^{2,3}$ In our case, the multiple organ cysts were excised and medical therapy was continued with albendazole (400 mg q12h) and praziquantel (20-75 mg/kg/day) for 1 year, due to extensive disease spread.

There is always a high chance of disease recurrence; therefore, continuation of medical treatment with an oral benzimidazole antihelmintic agent is recommended for at least 3 months after surgery. Pediatric patients often present with atypical symptoms, as in our case. Our patient had history of cough for the last 1 year and presented with acute respiratory distress. Earlier case reports of multiorgan involvement with primary dissemination have shown different approaches for disease management. Olmez et al and Kaya et al used surgical approach along with medical treatment for disseminated disease in children, as in our case. ${ }^{2,6}$ It has been observed that PAIR procedure is associated with greater efficacy and lower rates of complications than conventional surgery for the treatment of liver hydatid cysts. The procedure also has been used successfully in the treatment of other organs such as lungs and renal hydatid cysts. In the PAIR procedure, metronidazole can be used with a similar effect as hypertonic saline on the membrane of hydatid cyst. ${ }^{6}$

Anaphylactic reaction is a common complication of cystic hydatid disease and can occur due to trauma, intraoperatively or spontaneous nontraumatic leakage of cystic contents. Rupture of the cyst's contents evokes an allergic reaction that can range from minor hypersensitivity to a fatal anaphylactic shock. ${ }^{12}$

\section{Conclusion}

In endemic areas, hydatid disease with systemic dissemination, though rare, should be suspected while dealing with pediatric patients presenting with multiple cystic lesions. History, clinical data, and imaging could assist the physicians to make a definite diagnosis. Echinococcosis should be treated with either surgery or PAIR along with an oral benzimidazole antihelmintic agent to remove the cyst successfully without any complications and decrease the chances of recurrence.

\section{Funding}

None.

\section{Conflict of Interest}

None.

\section{References}

1 Ravis E, Theron A, Lecomte B, Gariboldi V. Pulmonary cyst embolism: a rare complication of hydatidosis. Eur J Cardiothorac Surg 2018;53(01):286-287

2 Iyigun O, Uysal S, Sancak R, et al. Multiple organ involvement hydatid cysts in a 2-year-old boy. J Trop Pediatr 2004;50(06): 374-376

3 Blanton R, Kliegman RM. Echinococcosis (Echinococcus granulosus and E. multilocularis). In: Behrman RE, Beds JH, eds. Nelson Textbook of Pediatrics. Sixteenth edition. Philadelphia: WB Saunders; 2008:1079-1081

4 Kurkcuoglu IC, Eroglu A, Karaoglanoglu N, Turkyilmaz A, Tekinbas C, Basoglu A. Surgical approach of pulmonary hydatidosis in childhood. Int J Clin Pract 2005;59(02):168-172

5 Gharbi HA, Hassine W, Brauner MW, Dupuch K. Ultrasound examination of the hydatic liver. Radiology 1981;139(02): 459-463

6 Mishra PK, Agrawal A, Joshi M, Sanghvi B, Gupta R, Parelkar SV. Minimal access surgery for multiorgan hydatid cysts. Afr J Paediatr Surg 2010;7(01):40-42

7 Kaya Z, Gursel T. A pediatric case of disseminated cystic echinococcosis successfully treated with mebendazole. Jpn J Infect Dis 2004;57(01):7-9

8 Lewall DB, McCorkell SJ. Hepatic echinococcal cysts: sonographic appearance and classification. Radiology 1985;155(03):773-775

9 Yuksel M, Demirpolat G, Sever A, Bakaris S, Bulbuloglu E, Elmas N. Hydatid disease involving some rare locations in the body: a pictorial essay. Korean J Radiol 2007;8(06):531-540

10 Safioleas M, Misiakos EP, Dosios T, Manti C, Lambrou P, Skalkeas G. Surgical treatment for lung hydatid disease. World J Surg 1999;23 (11):1181-1185

11 Prousalidis J, Tzardinoglou K, Sgouradis L, Katsohis C, Aletras H. Uncommon sites of hydatid disease. World J Surg 1998;22(01): $17-22$

12 Toumi O, Noomen F, Salem R, et al. Intraperitoneal rupture of hydatid cysts. Eur J Trauma Emerg Surg 2017;43(03):387-391 